\title{
Inducción de "tormenta de citocinas" en pacientes infectados con SARS- CoV-2 y desarrollo de COVID-19. ¿Tiene el tracto gastrointestinal alguna relación en la gravedad?
}

\author{
Induction of "Cytokine storm" in patients infected with SARS-CoV-2 and \\ development of COVID-19. Does the gastrointestinal tract any relation in severity?
}

Viviana Parra-Izquierdo, MD, ${ }^{*}$ Cristian Flórez-Sarmiento, MD, ${ }^{2}$ Consuelo Romero-Sánchez, PhD. ${ }^{3}$

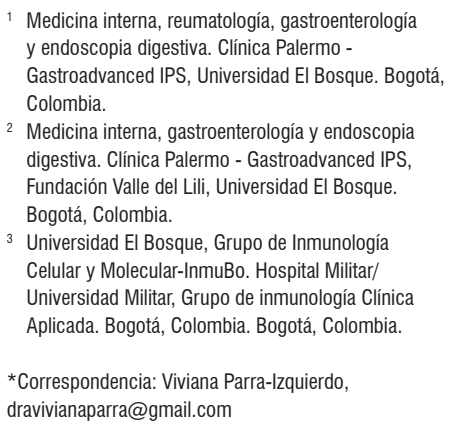

2 Medicina interna, gastroenterología y endoscopia digestiva. Clínica Palermo - Gastroadvanced IPS, Fundación Valle del Lili, Universidad El Bosque. Bogotá, Colombia.

3 Universidad El Bosque, Grupo de Inmunología Celular y Molecular-InmuBo. Hospital Militar/ Universidad Militar, Grupo de inmunología Clínica Aplicada. Bogotá, Colombia. Bogotá, Colombia.

${ }^{*}$ Correspondencia: Viviana Parra-Izquierdo, dravivianaparra@gmail.com

Fecha recibido: $\quad 09 / 04 / 20$

Fecha aceptado: 17/04/20

\begin{abstract}
Resumen
Los coronavirus son una extensa familia de virus; algunos pueden ser causa de diversas enfermedades humanas, que van desde el resfriado común hasta el síndrome respiratorio agudo severo. La enfermedad por coronavirus (COVID-19) es causada por el coronavirus zoonótico SARS-CoV-2, y comenzó en China en diciembre de 2019. En marzo de 2020, la Organización Mundial de la Salud (OMS) lo declaró una pandemia mundial. Esta enfermedad tiene un gran espectro de signos y sintomas, predominantemente se presenta con tos y fiebre, lo que resulta en una proporción de pacientes que desarrollan síndrome de dificultad respiratoria aguda (SDRA). Se ha planteado que la afectación pulmonar grave por el virus puede ser causada también por hiperinflamación y un cuadro similar a la linfohistiocitosis hemofagocítica $(\mathrm{HLH})$, el cual es un síndrome clínico provocado por una respuesta inadecuada del sistema inmunológico a un desencadenante, sea infeccioso, neoplásico, metabólico o reumatológico, que resulta en una reacción inflamatoria desproporcionada. Esta respuesta inmune altamente activada causa una liberación exagerada o tormenta de citocinas responsable del cuadro clínico. De allí la importancia de revisar el concepto actual y su desenlace en COVID-19. Recientemente se han publicado artículos en los que se sugiere que la presencia de síntomas gastrointestinales se ha asociado a COVID 19 con mayores indicadores de gravedad, lo que motiva al gastroenterólogo a explorar cómo el sistema digestivo podría impactar en esta hiperinflamación.
\end{abstract}

Palabras clave

Virus SARS, COVID-19, coronavirus, citocinas, tracto gastrointestinal.

\begin{abstract}
Coronaviruses are an extensive family of viruses, some of them can produce different kind of human diseases, ranging from the common cold to severe acute respiratory syndrome. Coronavirus disease (COVID-19) is caused by the SARS-CoV-2 zoonotic coronavirus and started in China in December 2019. In March 2020, the World Health Organization (WHO) declared it a global pandemic. This disease has a wide spectrum of signs and symptoms, predominantly presenting with cough and fever, resulting in a proportion of patients who develop acute respiratory distress syndrome (ARDS). It has been argued that severe lung involvement by the virus may also be caused by hyperinflammation and a condition similar to hemophagocytic lymphohistiocytosis $(H L H)$, which is a clinical syndrome caused by an inadequate response of the immune system to a trigger, whether infectious, neoplastic, metabolic, or rheumatologic, leading to a disproportionate inflammatory reaction. This activated immune response producing an exaggerated release or storm of cytokines responsible for this disease. There are recently publications suggesting that the presence of gastrointestinal symptoms is associated with COVID 19 disease with higher indicators of severity, which motivates the gastroenterologist to explore how the digestive system could impact this hyperinflammation.
\end{abstract}

\author{
Keywords \\ SARS Virus, COVID-19, Coronavirus, Cytokines, Gastrointestinal Tract.
}




\section{INTRODUCCIÓN}

En diciembre de 2019, un nuevo coronavirus (coronavirus 2 del síndrome respiratorio agudo severo [SARS-CoV-2]) surgió en China, y se ha extendido a nivel mundial, lo que ocasionó una pandemia (1). Este virus, el SARS-CoV-2, se transmite principalmente por medio de gotas en el aire, pero también hay una eliminación viral en las heces, lo cual plantea otra vía de trasmisión como lo es la fecal-oral (2). Esta enfermedad tiene un gran espectro de signos y síntomas, predominantemente se presenta con tos y fiebre, lo que resulta en una proporción de pacientes que desarrollan síndrome de dificultad respiratoria aguda (SDRA) (3); también puede causar síntomas gastrointestinales como diarrea, náuseas, vómitos y dolor abdominal, los cuales se han reportado entre el 11,5\%-32\% de los pacientes (4). Sin embargo, llama la atención porque algunos pacientes desarrollan una enfermedad tan grave con altas tasas de mortalidad (5). Se ha planteado que la afectación pulmonar grave del virus puede ser causada también por hiperinflamación y un cuadro similar a la linfohistiocitosis hemofagocítica $(\mathrm{HLH})$, el cual es un síndrome clínico provocado por una respuesta inadecuada del sistema inmunológico a un desencadenante, sea infeccioso, neoplásico, reumatológico o metabólico, lo que resulta en una reacción inflamatoria exagerada (6). La HLH no es una única enfermedad, sino un síndrome clínico asociado a gran variedad de causas subyacentes que conducen al mismo fenotipo inflamatorio característico. Esta respuesta inmune altamente activada causa una liberación exagerada o una "tormenta de citocinas” responsable del cuadro clínico (7).

\section{COVID-19 Y LA TORMENTA DE CITOCINAS}

Huang y colaboradores describen las principales características clínicas de 41 pacientes infectados con el nuevo SARSCoV-2 en China, estudio en el que se incluyó la evaluación de citocinas y algunas quimiocinas con el fin de caracterizar el efecto del coronavirus sobre su producción (8).

Se evaluaron las concentraciones plasmáticas de un amplio panel (interleucinas (IL)-1B, IL-1RA, IL-2, IL-4, IL-5, IL-6, IL-7, IL-8 (también conocido como CXCL8), IL-9, IL-10, IL-12p70, IL-13, IL-15, IL-17A, eotaxina (también conocido como CCL11), FGF2 básico (fibroblast growth factor), CSF-G (colony stimulating factor granulocyte), CSF-GM (colony stimulating factor granulocyte/ monocyte), IFN $\gamma$ (interferon gamma), IP10 (interferon gamma-induced protein 10-CXCL10), MCP1 (monocyte chemoattractant protein 1-CCL2), MIP1A (macrophage inflammatory protein 1-alpha-CCL3), MIP1B (macrophage inflammatory protein 1-alpha CCL4), PDGFB (plateletderived growth factor subunit B), RANTES (CCL5), TNF $\alpha$ (tumor necrosis factor- $\alpha$ ) y VEGFA (vascular endothelial growth factor A). El método utilizado incluyó un sistema múltiple que evaluó 27 citocinas simultáneamente (BioPlex 200, Bio-Rad, Hercules, CA, EE. UU.). Las muestras de plasma de cuatro adultos sanos se usaron como controles para la comparación. El tiempo medio desde que se transfirieron a un hospital designado para la recolección de muestras de sangre fue de 4 días (IQR 2-5) (8).

Los resultados demostraron altas concentraciones de IL-1B, IL-1RA, IL-7, IL-8, IL-9, IL-10, FGFb, CSF-G, CSF-GM, IFN $\gamma$, IP10, MCP1, MIP1A, MIP1B, PDGF, TNF $\alpha$ y VEGF, independiente del ingreso a la unidad de cuidados intensivos (UCI) en comparación con individuos adultos sanos. Los niveles plasmáticos de IL-5, IL-12p70, IL-15, eotaxina y RANTES fueron similares entre los adultos sanos y pacientes con COVID-19. De otra manera, al comparar pacientes que ingresaron a la UCI y los que no necesitaron el ingreso, las concentraciones plasmáticas de IL-2, IL-7, IL-10, CSF-G, IP10, MCP1, MIP1A y TNF $\alpha$ fueron mayores en pacientes que ingresaron a la UCI (Figura 1) (8).

La fisiopatología de la alta patogenicidad para SARS$\mathrm{CoV}$ o MERS-CoV no ha sido completamente entendida. Los primeros estudios han demostrado que hay un aumento excesivo de citocinas proinflamatorias en suero (p. ej., IL-1B, IL-6, IL-12, IFN $\gamma$, IP10 y MCP1), y esto se ha asociado con inflamación pulmonar y compromiso pulmonar grave en pacientes con SARS (Severe Acute Respiratory Syndrome) (9). La primera evidencia sugiere que un subgrupo de pacientes con COVID-19 grave podría presentar un "síndrome de tormenta de citocinas" $(8,10)$.

El término tormenta de citocinas ha captado la atención de la comunidad científica en general, y aunque el concepto corresponde a una liberación excesiva o incontrolada de citocinas proinflamatorias, el concepto y las consecuencias biológicas de la sobreproducción de citocinas no son claras. Las tormentas de citocinas están asociadas con una amplia variedad de enfermedades infecciosas y no infecciosas (2). En 1993, un grupo en Boston, quizás consciente de la reciente guerra de la Tormenta del Desierto, acuñó el término tormenta de citocinas para describir sus observaciones sobre la enfermedad de injerto contra huésped (EICH) (4). El término apareció luego en 2002 como una descripción del mecanismo de la enfermedad en la pancreatitis $(11,12)$. Al igual que con la $\mathrm{EICH}$, la idea era más antigua que el término adecuadamente descriptivo, con una citocina pro y antiinflamatoria incriminada en esta condición en 1992 y 1997, respectivamente.

Posteriormente el primer uso del término para describir el mecanismo de una enfermedad infecciosa probablemente se observó en 2003, en la encefalopatía por influenza seguido de la aplicación al virus de la viruela y al de la gripe 


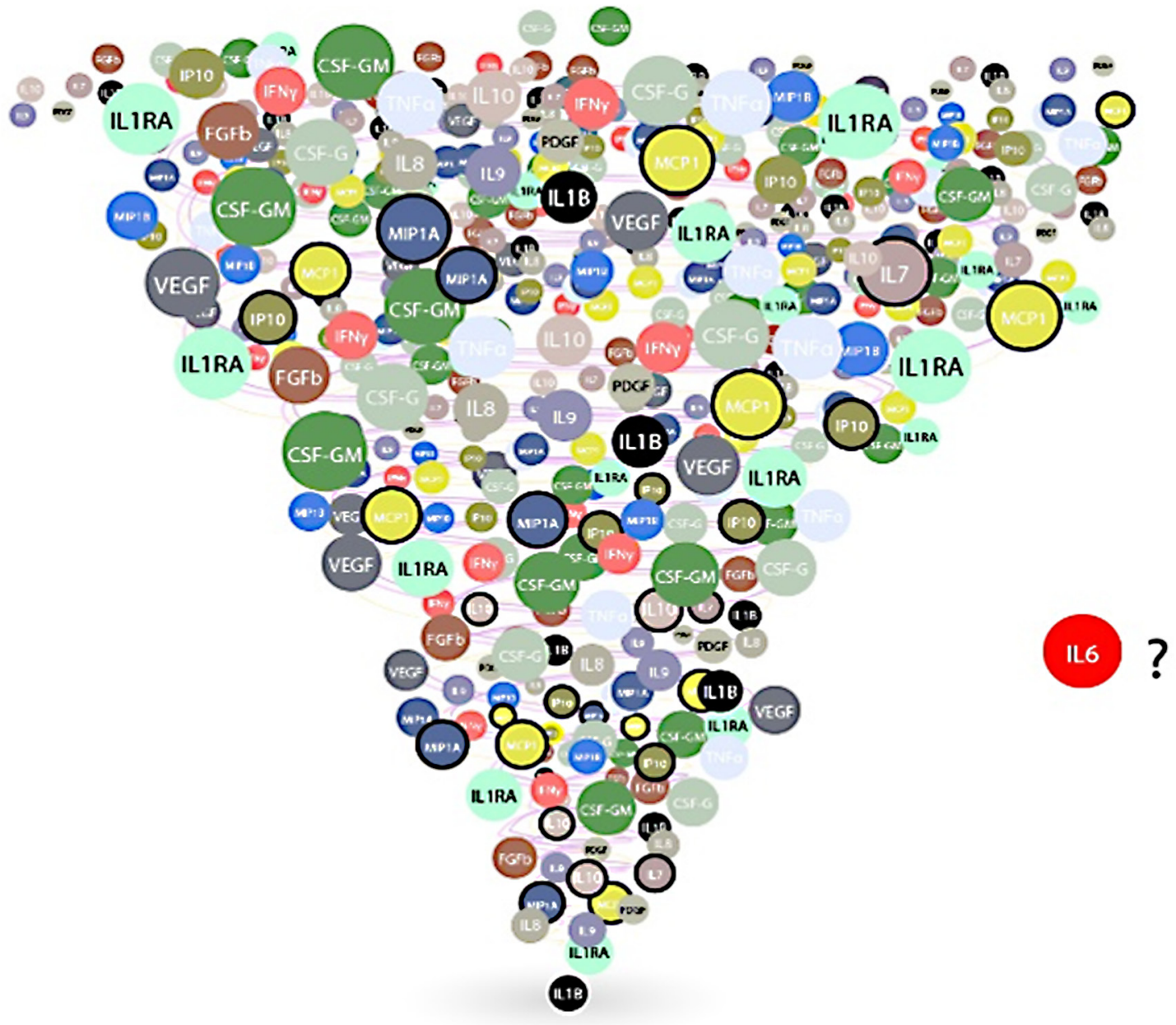

Figura 1. Tormenta de citocinas en COVID-19 (1). Los resultados demostraron altas concentraciones de IL-1B, IL-1RA, IL-7, IL-8, IL-9, IL10, FGFb, CSF-G, CSF-GM, IFN $\gamma$, IP10, MCP1, MIP1A, MIP1B, PDGF, TNF $\alpha$ y VEGF, independiente del ingreso a la UCI. Los pacientes que ingresaron a la UCI presentaron las concentraciones plasmáticas más altas de IL-2, IL-7, IL-10, CSF-G, IP10, MCP1, MIP1A y TNF (círculos con reborde). En este grupo no encontraron IL-6. Diseñado por Juan Báez R.

$\mathrm{H}_{5} \mathrm{~N}_{1}$ (13). Los síndromes de tormenta de citocinas son un grupo de trastornos que representan una variedad de causas inflamatorias. Los síntomas principales de una tormenta de citocinas son fiebre alta, inflamación y enrojecimiento, fatiga extrema y náuseas. En algunos casos, la reacción inmune puede ser fatal, como en COVID-19.

En la tormenta de citocinas posterior a una infección, la inflamación comienza cuando las células del sistema inmune innato reconocen una estructura del microorganismo (virus en este caso) denominado patrón molecular asociado al patógeno (PAMP), que posee el organismo invasor; sin embargo, hay que aclarar que compuestos propios derivados de nuestro organismo asociados al daño pueden activar igualmente la inmunidad natural (DAMP).
Los receptores en las células fagocíticas del huésped que reconocen estos PAMP o DAMP se conocen como receptores de patrones reconocimiento (PRR), de los cuales hay diferentes categorías; tal vez los que más conocemos son los Toll-like receptor (TLR), también están los Nod-like receptors (NLR), entre otros. Estos receptores se encuentran en la superficie de las membranas, en el citosol o de forma soluble (14).

Una vez que se une el PAM al receptor PRR, se activa y se produce una cascada de señalización, que da como resultado la expresión de citocinas proinflamatorias, principalmente. Esta producción de citocinas es muy importante porque desempeña un papel vital en las primeras etapas de la inflamación. Durante la primera fase de la infección, estas 
moléculas proteicas actúan como señales para el sistema inmune, regulando la duración y la gravedad de la respuesta inmune frente al daño o a la infección. Dependiendo de la citocina específica que ha sido secretada, su función puede ser activar (proinflamatorio) o regular (antiinflamatorio) la respuesta del huésped (15).

Diferentes tipos de células pueden secretar estas citocinas quimiotácticas, incluidas las células fagocíticas, como los macrófagos y los neutrófilos, pero no podemos olvidar que las células endoteliales son responsables de más de la mitad de todas las citocinas producidas. Las quimiocinas activan las moléculas de adhesión, lo que facilita la migración (16). Posteriormente, las células ruedan a lo largo del endotelio, atraídas por un gradiente químico generado por las quimiocinas hacia el sitio de inflamación, donde transmigran a través de uniones celulares en el tejido infectado. Finalmente, posterior a su activación, la respuesta inflamatoria debe ser regulada para prevenir una inflamación sistémica deletérea también conocida como tormenta de citocinas; para esto, una serie de citocinas con propiedades antiinflamatorias son responsables de controlar, como la IL-10 y el factor de crecimiento transformante beta (TGF-B) (Figura 2).

En particular, la IL-1 es un importante mediador de inflamación local y sistémica, generada por infecciones virales que afectan el pulmón, lo que induce inflamación tisular, fiebre y fibrosis $(11,17)$. Los macrófagos activados por el SARS-CoV-2 están directamente relacionados con la fibrosis, dado que ellos son los responsables de eliminar a través de su actividad fagocítica los detritus derivados de las células y tejidos muertos (DAMP), evitando que estos se unan a PRR e induzca inflamación; estas células migran atraídas por la IL-8 generada desde el sitio de inflamación (Figura 2).

Se ha descrito que el SARS-CoV-2 se une a TLR2, TLR3 o TLR4, induciendo una gran cascada de citocinas dentro de las cuales está la IL-1B, así como la IL-6; por esta razón, la supresión de estas citocinas ha sido considerada un tratamiento efectivo para muchas patologías inflamatorias (18). La producción de citocinas proinflamatorias se correlacionan con la tasa de replicación del virus SARS-CoV-2, y de

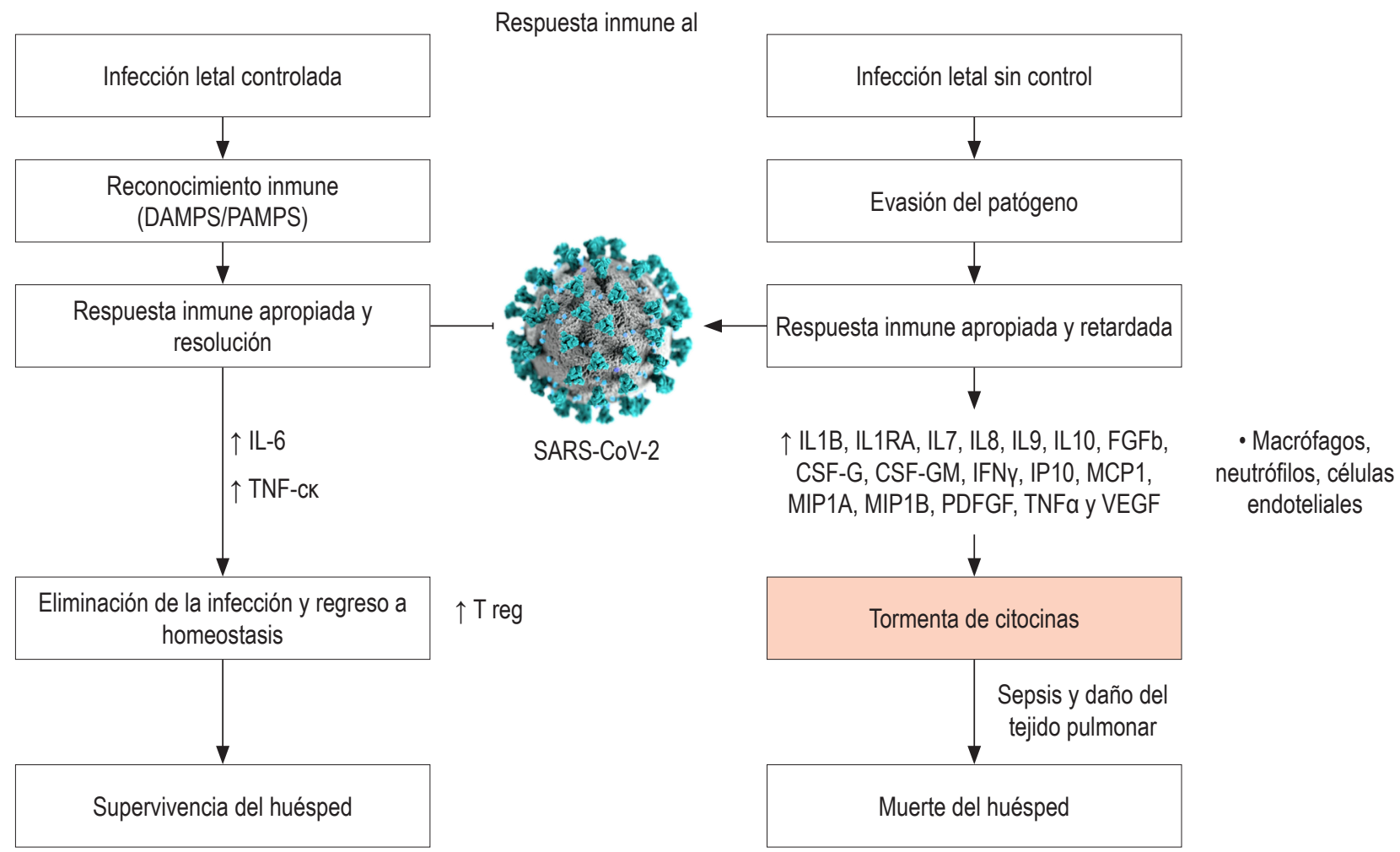

Figura 2. Respuesta frente a una infección viral. El huésped reconoce las estructuras del patógeno (PAMS), que conduce al reclutamiento celular y a una respuesta de citocinas proinflamatorias que incluyen principalmente la IL-6 y el TNF. Esta respuesta inflamatoria convencionalmente conduce a la eliminación del patógeno, lo que permite el retorno a la homeostasis inmune y la supervivencia del huésped infectado. En algunas infecciones, el reconocimiento por el sistema inmune se retrasa y/o evade, lo que provoca una respuesta retardada y/o inapropiada. Esto puede permitir que el patógeno prolifere, lo que desencadena la hipercitocinemia o tormenta de citocinas, conllevando a daño tisular y potencialmente a la muerte del huésped $(1,15)$. Diseñado por Juan Báez R. 
manera intuitiva se considera que los antagonistas de IL-1, como la IL1Ra, la IL37 o IL38, pueden proveer regulación sistémica (19).

Se ha encontrado que diversos virus patógenos (p. ej., influenza A y ahora el SARS-COV-2) y bacterias (como Francisella tularensis) inducen la tormenta de citocinas o la hipercitocinemia (20). Estos patógenos inducen al quiebre del delicado equilibrio de una respuesta inflamatoria adecuada, y se inclinan a un desenlace destructivo dada la inducción de la retroalimentación positiva no controlada en las células inmunes y la regulación positiva de producción de marcadores proinflamatorios, en particular las citocinas TNF, IL-1, IL-8 e IL-6. Esto pronto produce síntomas tales como hipotensión, fiebre y edema, eventualmente puede causar disfunción orgánica y muerte.

Un perfil de citocinas asociado con el síndrome respiratorio severo se asoció con la gravedad de COVID-19, caracterizada por un aumento de IL-2, IL-7, CFS-GM, INF IP10 $\gamma$, MCP1 $\alpha$ y TNF $\alpha$ (21). Por otro lado, en la infección por MERS-CoV se reportaron mayores concentraciones de citocinas proinflamatorias como el IFN $\gamma$, TNFa, IL-15 y IL-17 (20). Específicamente, en 41 sujetos con COVID-19 de la región de Wuhan, se observó que los pacientes infectados presentaron en plasma altas cantidades de IL-1B, IFN $\gamma$, IP10 y MCP1, lo que probablemente conduce a la activación del perfil de célula T-helper-1 (Th1) (8).

De manera particular, los pacientes que requirieron ingreso en la UCI tenían mayores concentraciones de CSF-G, IP10, MCP1, MIP1A, y TNF $\alpha$ que aquellos que no ingresaron a la UCI (Figura 1); esto sugiere que la tormenta de citocinas estaba asociada con la gravedad de la enfermedad (8). Como dato importante, la infección por SARS-CoV-2 también inició con una mayor secreción de citocinas del perfil T-helper-2 (Th2) (p. ej., IL-4 e IL-10), las cuales suprimen la inflamación, lo que realmente difiere de la infección por SARS-CoV (9). Se ha descrito que la producción sistémica de IL-10 después del inicio de una tormenta de citocinas es un marcador de una respuesta antiinflamatoria denominado inmunoparálisis, ya que está asociado con una regulación a la baja de la función de neutrófilos y monocitos en la circulación (22). Esto podría ser conceptualmente beneficioso en el control sistémico de las respuestas a infecciones locales; sin embargo, se ha sugerido que los pacientes que sobreviven a la tormenta inicial de citocinas, posteriormente pueden morir si no se recuperan de la inmunoparálisis (23).

No es clara esta dualidad, por lo que los autores plantean la necesidad de insistir en la caracterización de las respuestas Th1 y Th2 en la infección por SARS-CoV-2, y poder acercarse a dilucidar la real patogénesis de COVID-19. Los autores proponen que los estudios de autopsia o biopsia serían la clave para entender la enfermedad (8).

En resumen, la inflamación asociada con una tormenta de citocinas comienza en el sitio local y se extiende por todo el organismo a través de la circulación sistémica. Estas respuestas a menudo ocurren con el gasto de la función del órgano local, particularmente cuando el edema tisular provoca un aumento en las presiones extravasculares y una reducción en la perfusión del tejido. Los procesos de reparación compensatorios se inician poco después de comenzar la inflamación y, en muchos casos, el proceso de reparación restaura por completo la función de tejidos y órganos. Cuando la inflamación grave o el agente etiológico primario que desencadena la inflamación (SARS-CoV-2) daña las estructuras locales del tejido, la reparación ocurre con fibrosis, lo que puede provocar disfunción orgánica persistente. La lesión pulmonar aguda es una consecuencia común de una tormenta de citocinas, como la descrita en COVID-19 en el ambiente alveolar y en la circulación sistémica. Esta respuesta se caracteriza por un infiltrado inflamatorio mononuclear y neutrofílico agudo, seguido de una fase fibroproliferativa crónica marcada por el depósito progresivo de colágeno en el pulmón. La lesión pulmonar inducida por patógenos puede progresar hacia lesión pulmonar aguda o, su forma más grave, síndrome de dificultad respiratoria aguda (SDRA), llevando en muchos casos a desenlaces fatales (11) (Figura 3).

Teniendo en cuenta lo anterior, con la evidencia actual acumulada, se sugiere que un subgrupo de pacientes con COVID-19 grave podrían tener un síndrome de tormenta de citocinas con un perfil de citocinas que se asemeja a $\mathrm{HLH}$, por lo que se sugiere el tratamiento de esta hiperinflamación dado que la falla respiratoria del SDRA es la principal causa de mortalidad.

Predictores de mortalidad de un estudio reciente retrospectivo multicéntrico de 150 casos confirmados de COVID-19 en Wuhan, China, incluyeron ferritina elevada con medias de $1297,6 \mathrm{ng} / \mathrm{mL}$ en no sobrevivientes frente a $614 \mathrm{ng} / \mathrm{mL}$ en sobrevivientes; $p<0,001)$ e IL-6 ( $p$ $<0,0001)$, lo que sugiere que la mortalidad puede deberse a una hiperinflamación dirigida por este virus. Por tal motivo, se considera que a todos los pacientes con COVID-19 grave se les debería evaluar estos biomarcadores de hiperinflamación, y que hallazgos como el aumento de ferritina, la disminución de conteos plaquetarios, reactantes de fase aguda o dímero $\mathrm{D}$ elevados asociados al HScore (puntaje de gravedad) elevado, este último otra herramienta que se utiliza en HLH (24), se podrían identificar en el subgrupo de pacientes para quienes la inmunosupresión podría mejorar o disminuir la mortalidad (25). 


\section{GRAVEDAD Y SÍNTOMAS GASTROINTESTINALES}

La presencia de síntomas gastrointestinales ha empezado a tener un papel muy importante, ya que se han asociado a COVID 19 con mayores indicadores de gravedad, lo que motiva al gastroenterólogo a explorar cómo el sistema digestivo podría impactar en esta hiperinflamación (Figura 3). Estudios previos con el SARS indicaron que el Coronavirus puede tener un tropismo por el tracto gastrointestinal $(2,26)$. Este hallazgo tiene implicaciones importantes en el manejo de la enfermedad, la transmi- sión y el control de la infección; bajo el contexto de esta tormenta de citocinas que se produce en COVID-19, el tracto gastrointestinal ha demostrado que también tiene un protagonismo dado que cuando la mucosa intestinal está bajo estrés se produce un sobrecrecimiento bacteriano y un inicio progresivo de translocación bacteriana. Esto produce isquemia, la cual altera las vellosidades intestinales, con una alteración de la permeabilidad y la función de la barrera intestinal que favorece, de esta forma, aún más la translocación. De esta manera se produce una disfunción silenciosa y, en ocasiones, imperceptible, en forma secun-

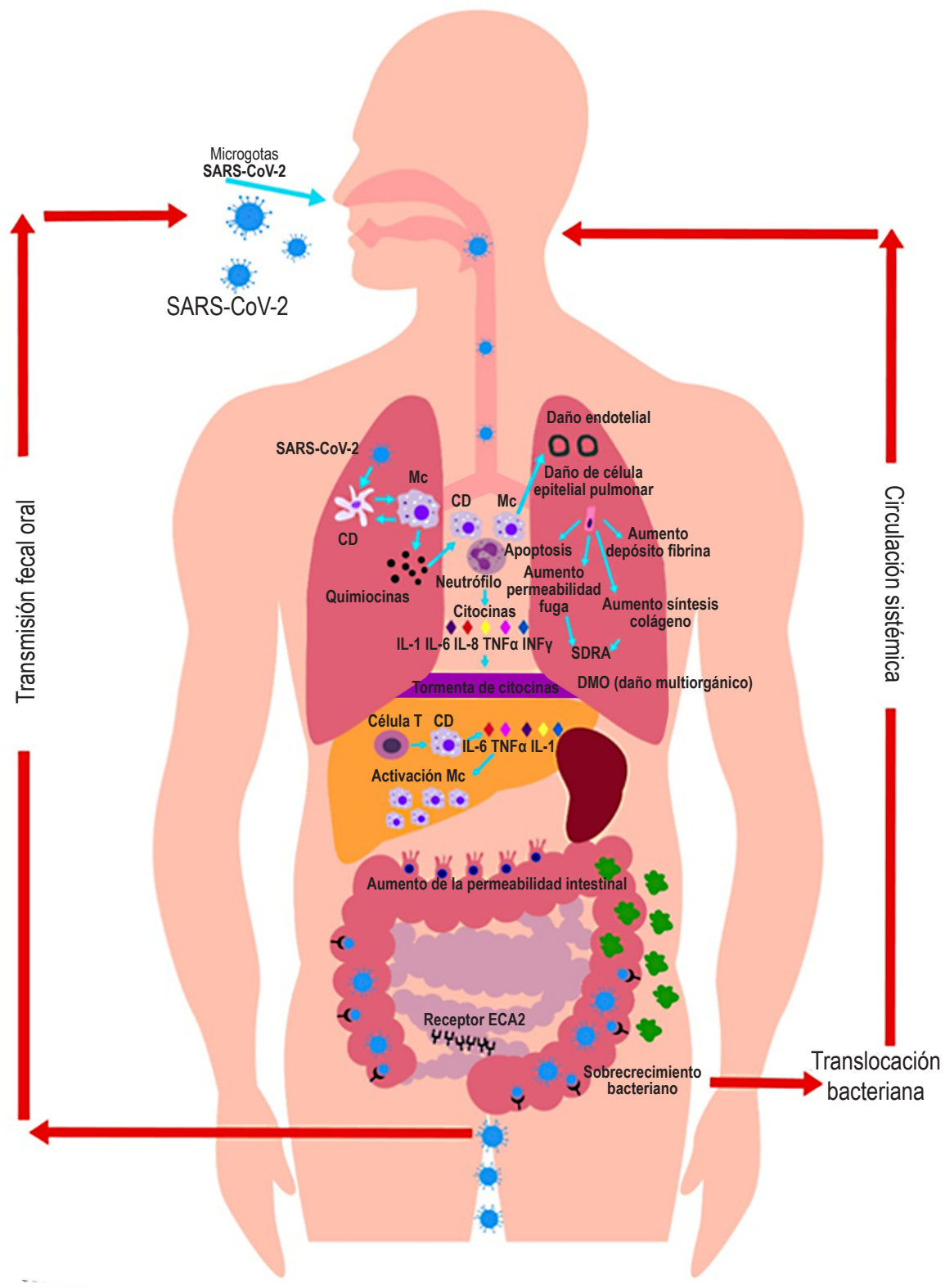

Figura 3. Mecanismos de transmisión y afectación en órganos blanco. Se presenta una afectación pulmonar grave por el virus causada por la hiperinflamación y un cuadro similar a la HLH. Esta respuesta inmune altamente activada causa una liberación exagerada o tormenta de citocinas. Además del contagio por gotas y aerosoles, se ha documentado transmisión fecal-oral, sustentando la presencia de síntomas gastrointestinales en COVID-19. CD: célula dendrítica; Mc: macrófagos. Diseñado por Dra. Viviana Parra-Izquierdo; graficado por Ana María Ocaña. 
daria al paso de bacterias, endotoxinas y mediadores inflamatorios, que superan el filtro hepático y alcanzan la circulación sistémica; por tanto, el intestino desempeña una importante función en la progresión de la sepsis hacia el fallo múltiple de órganos, donde se altera la integridad del intestino por un incremento de la apoptosis y de la composición del moco intestinal, a lo que se adiciona la toxicidad a distancia. Estas condiciones favorecen que se perpetúe la respuesta inflamatoria sistémica en pacientes críticamente enfermos (27-29).

En COVID-19, al evidenciar la presencia de síntomas gastrointestinales, se producen cuestionamientos sobre si este compromiso intestinal podría asociarse a un compromiso más grave de esta enfermedad.

En un metanálisis de 60 estudios, compuesto por 4243 pacientes, la prevalencia combinada de todos los síntomas gastrointestinales fue del 17,6 \% (intervalo de confianza [IC] del $95 \%, 12,3 \%-24,5 \%)$. El 11,8 \% de los pacientes con COVID-19 no grave tenían síntomas gastrointestinales (IC del $95 \%$, 4,1 \%-29,1 \%), mientras que el 17,1 \% de los pacientes con COVID-19 grave tenían síntomas gastrointestinales (IC del 95 \%, 6,9 \%-36,7 \%). En el metaanálisis, la prevalencia combinada de muestras de heces que fueron positivas para el ARN del virus fue del 48,1 \% (IC del $95 \%$, 38,3 \%-57,9 \%). De estas muestras recolectadas, el 70,3\% después de la desaparición del virus de las muestras respiratorias dieron positivo para el virus (IC del $95 \%$, 49,6\%$85,1 \%)$. Se detectó ARN viral en heces en $38,5 \%$ y $8,7 \%$ entre aquellos con y sin diarrea, respectivamente $(p=0,02)$. La mediana de la carga viral fecal fue de $5,1 \log 10 \mathrm{cpm}$ en pacientes con diarrea frente a $3,9 \log 10 \mathrm{cpm}$ en pacientes sin diarrea $(p=0,06)(30)$.

Otro estudio incluyó a 651 pacientes con COVID-19 entre el 17 de enero de 2020 al 8 de febrero de 2020 en la provincia de Zhejiang, de los cuales $74(11,4 \%)$ presentaron al menos un síntoma del tracto gastrointestinal (náuseas, vómito y diarrea), siendo mayor que los datos anteriores de Wuhan. Curiosamente, la tasa de enfermedad hepática crónica en este grupo fue del 10,81 \%, lo cual es significativamente mayor que la del $2,95 \%$ en aquellos sin síntomas gastrointestinales $(p=0,004)$. Algo muy importante fue que también aumentó notablemente la gravedad con fiebre, SDRA, lesión hepática y choque en el grupo con síntomas gastrointestinales frente a aquellos sin síntomas gastrointestinales $(22,97 \%$ frente a $8,14 \%, p<0,001)(31)$.

Teniendo en cuenta todas estas alteraciones inmunológicas y esta hiperinflamación, se han planteado múltiples estrategias terapéuticas enfocadas en contener el daño pulmonar, el cual es el que está siendo asociado al aumento de la mortalidad. Durante pandemias previas por SARS y MERS, los corticoesteroides no fueron dados de rutina (32); sin embargo, otras herramientas de inmuno- supresión podrían ayudar a controlar la hiperinflamación. Existen datos de estudios aleatorizados controlados con el uso de bloqueadores de anti-IL-1 (anakinra) en sepsis que muestran supervivencia en sepsis con hiperinflamación sin incrementar los eventos adversos (33); también hay un estudio aleatorizado controlado con el uso de tocilizumab, un bloqueador del receptor de IL-6 para el síndrome de tormenta de citocinas en pacientes con COVID-19 con daño pulmonar, donde no se recomendó el uso rutinario de corticoides (34). Actualmente, entre las opciones terapéuticas que podrían plantearse incluso podría no descartarse totalmente el uso de esteroides, también la inmunoglobulina intravenosa, el bloqueo selectivo de citocinas (p. ej., anakinra o tocilizumab) y la inhibición de las vías JAK.

\section{CONCLUSIÓN}

El entendimiento de este fenómeno de la producción de la denominada tormenta de citocinas y la detección de un rápido empeoramiento de COVID-19 con manifestaciones sistémicas, respiratorias, y la evaluación de algunas de las citocinas descritas, así como los niveles de IL-1B, IL-6 y dímero $\mathrm{D}$, pueden representar un escenario útil para la identificación de los respondedores ideales a terapias dirigidas. No se debe olvidar que el intestino dejó de ser un órgano exclusivo para la nutrición y desempeña funciones inmunológicas, y que, en esta pandemia, el compromiso gastrointestinal está comenzado a tener un protagonismo en pacientes con enfermedad grave. Más estudios y ensayos clínicos que están actualmente en curso nos ayudarán a mejorar la elegibilidad para una intervención más agresiva.

\section{Fuente de financiación}

Se declara que no existieron apoyos financieros para la realización y el análisis de este artículo. Este artículo no ha sido presentado previamente en ninguna publicación.

\section{REFERENCIAS}

1. WHO [Internet]. WHO Director-General's opening remarks at the media briefing on COVID-19 - 11 March 2020. 2020 [citado 31 de marzo de 2020]. Disponible en: https://www.who.int/dg/speeches/detail/who-directorgeneral-s-opening-remarks-at-the-media-briefing-on-covid19---11-march-2020

2. Gu J, Han B, Wang J. COVID-19: Gastrointestinal manifestations and potential fecal-oral transmission. Gastroenterology. 2020. https://doi.org/10.1053/j.gastro.2020.02.054

3. Cascella M, Rajnik M, Cuomo A, et al. Features, Evaluation and Treatment Corona-virus (COVID-19) [actualizado 6 de abril de 2020]. En: StatPearls [Internet]. Treasure Is-land 
(FL): StatPearls Publishing; 2020. Disponible en: https:// www.ncbi.nlm.nih.gov/books/NBK554776/

4. Xiao F, Tang M, Zheng X, Liu Y, Li X, Shan H. Evidence for Gastrointestinal In-fection of SARS-CoV-2. Gastroenterology. 2020. pii: S0016-5085(20)30282-1. https://doi.org/10.1053/j.gastro.2020.02.055

5. Davenne E, Giot JB, Huynen P. [Coronavirus and COVID19 : focus on a galop-ping pandemic]. Rev Med Liege. 2020;75(4):218-225.

6. Dapena Díaz JL, Díaz de Heredia Rubio C, Bastida Vila P, Llort Sales A, Elorza Alvarez I, Olivé Oliveras T, et al. [Haemophagocytic syndrome: A common pathogenic mechanism of various aetiologies]. An Pediatr (Barc). 2009;71(2):110-6. https://doi.org/10.1016/j. anpedi.2009.04.008

7. Buda P, Gietka P, Wieteska-Klimczak A, Ksiazyk J. [Secondary hemophagocytic syndromes]. Wiad Lek. 2013;66(2 Pt 2):153-63.

8. Huang C, Wang Y, Li X, Ren L, Zhao J, Hu Y, et al. Clinical features of patients infected with 2019 novel coronavirus in Wuhan, China. Lancet. 2020;395(10223):497-506. https://doi.org/10.1016/S0140-6736(20)30183-5

9. Wong CK, Lam CW, Wu AK, Ip WK, Lee NL, Chan IH, et al. Plasmainflammato-ry cytokines and chemokines in severe acute respiratory syndrome. Clin Exp Immunol. 2004;136(1):95103. https://doi.org/10.1111/j.1365-2249.2004.02415.x

10. Mehta P, McAuley DF, Brown M, Sanchez E, Tattersall RS, Manson JJ, et al. COVID-19: consider cytokine storm syndromes and immunosuppression. Lancet. 2020;395(10229):1033-1034. https://doi.org/10.1016/ S0140-6736(20)30628-0

11. Tisoncik JR, Korth MJ, Simmons CP, Farrar J, Martin TR, Katze MG. Into the eye of the cytokine storm. Microbiol Mol Biol Rev. 2012;76(1):16-32. https://doi.org/10.1128/ MMBR.05015-11

12. Makhija R, Kingsnorth AN. Cytokine storm in acute pancreatitis. J Hepatobiliary Pancreat Surg. 2002;9(4):401-10. https://doi.org/10.1007/s005340200049

13. Ferrara JL. Cytokine dysregulation as a mechanism of graft versus host disease. Curr Opin Immunol. 1993;5(5):794-9. https://doi.org/10.1016/0952-7915(93)90139-j

14. Barton GM. A calculated response: control of inflammation by the innate immune system. J Clin Invest. 2008;118(2):413-20. https://doi.org/10.1172/JCI34431

15. D>Elia RV, Harrison K, Oyston PC, Lukaszewski RA, Clark GC. Targeting the «cy-tokine storm» for therapeutic benefit. Clin Vaccine Immunol. 2013;20(3):319-27. https://doi. org/10.1128/CVI.00636-12

16. Murdoch C, Finn A. Chemokine receptors and their role in inflammation and infec-tious diseases. Blood. 2000;95(10):3032-43.

17. Kritas SK, Ronconi G, Caraffa A, Gallenga CE, Ross $\mathrm{R}$, Conti P. Mast cells con-tribute to coronavirus-induced inflammation: new anti-inflammatory strategy. J Biol Regul Homeost Agents. 2020;34(1). https://doi. org/10.23812/20-Editorial-Kritas
18. Conti P, Ronconi G, Caraffa A, Gallenga CE, Ross R, Frydas I, et al. Induction of pro-inflammatory cytokines (IL-1 and IL-6) and lung inflammation by Coronavirus-19 (COVI-19 or SARS-CoV-2): anti-inflammatory strategies. J Biol Regul Homeost Agents. 2020 Mar 14;34(2). pii: 1. https://doi. org/10.23812/CONTI-E

19. Marchetti C, Swartzwelter B, Koenders MI, Azam T, Tengesdal IW, Powers N, et al. NLRP3 inflammasome inhibitor OLT1177 suppresses joint inflammation in murine models of acute arthritis. Arthritis Res Ther. 2018;20(1):169. https://doi.org/10.1186/s13075-018-1664-2

20. Us D. [Cytokine storm in avian influenza]. Mikrobiyol Bul. 2008;42(2):365-80.

21. Mahallawi WH, Khabour OF, Zhang Q Makhdoum HM, Suliman BA. MERS-CoV infection in humans is associated with a pro-inflammatory Th1 and Th17 cytokine profile. Cytokine. 2018;104:8-13. https://doi.org/10.1016/j. cyto.2018.01.025

22. Fowler AA, Fisher BJ, Centor RM, Carchman RA. Development of the adult respir-atory distress syndrome: progressive alteration of neutrophil chemotactic and secretory pro-cesses. Am J Pathol. 1984;116(3):427-35.

23. Munford RS, Pugin J. Normal responses to injury prevent systemic inflammation and can be immunosuppressive. Am J Respir Crit Care Med. 2001;163(2):316-21. https://doi. org/10.1164/ajrccm.163.2.2007102

24. Fardet L, Galicier L, Lambotte O, Marzac C, Aumont C, Chahwan D, et al. Devel-opment and validation of the HScore, a score for the diagnosis of reactive hemophagocytic syndrome. Arthritis Rheumatol. 2014;66(9):2613-20. https://doi.org/10.1002/art.38690.

25. Ruan Q, Yang K, Wang W, Jiang L, Song J. Clinical predictors of mortality due to COVID-19 based on an analysis of data of 150 patients from Wuhan, China. Intensive Care Med. 2020. https://doi.org/10.1007/s00134-020-05991-x

26. Zheng F, Liao C, Fan QH, Chen HB, Zhao XG, Xie ZG, et al. Clinical Characteris-tics of Children with Coronavirus Disease 2019 in Hubei, China. CurrMed Sci. 2020;40(2):275280. https://doi.org/10.1007/s11596-020-2172-6

27. Shimizu K, Ogura H, Hamasaki T, Goto M, Tasaki O, Asahara $\mathrm{T}$, et al. Altered gut flora are associated with septic complications and death in critically ill patients with systemic inflammatory response syndrome. Dig Dis Sci. 2011;56(4):1171-7. https://doi.org/10.1007/s10620-010-1418-8

28. Fasano A. Zonulin and its regulation of intestinal barrier function: the biological door to inflammation, autoimmunity, and cancer. Physiol Rev. 2011;91(1):151-75. https:// doi.org/10.1152/physrev.00003.2008

29. Salvo-Romero E, Cotoner M del CA, Camacho CP, CasadoBedmar M, Vicario M. Función barrera intestinal y su implicación en enfermedades digestivas. Rev Esp Enferm Dig. 2015;107(11):686-96.

30. Cheung KS, Hung IF, Chan PP, Lung KC, Tso E, Liu R, et al. Gastrointestinal Manifestations of SARS-CoV-2 Infection and Virus Load in Fecal Samples from the Hong Kong Cohort and Systematic Review and Meta-analysis. 
Gastroenterology. 2020. pii:S0016-5085(20)30448-0. https://doi.org/10.1053/j.gastro.2020.03.065

31. Jin X, Lian JS, Hu JH, Gao J, Zheng L, Zhang YM, et al. Epidemiological, clinical and virological characteristics of 74 cases of coronavirus-infected disease 2019 (COVID-19) with gastrointestinal symptoms. Gut. 2020. pii: gutjnl-2020-320926. https://doi.org/10.1136/gutjnl-2020-320926

32. Russell CD, Millar JE, Baillie JK. Clinical evidence does not support corticosteroid treatment for 2019-nCoV lung injury. Lancet. 2020;395(10223):473-475. https://doi. org/10.1016/S0140-6736(20)30317-2
33. Shakoory B, Carcillo JA, Chatham WW, Amdur RL, Zhao $\mathrm{H}$, Dinarello CA, et al. Interleukin-1 Receptor Blockade Is Associated With Reduced Mortality in Sepsis Patients With Features of Macrophage Activation Syndrome: Reanalysis of a Prior Phase III Trial. Crit Care Med. 2016;44(2):27581. https://doi.org./10.1097/CCM.0000000000001402

34. Chinese Clinical Trial Register (ChiCTR) [Internet]. The world health organization international clinical trials registered organization registered platform [citado 9 de abril de 2020]. Disponible en: http://www.chictr.org.cn/showprojen.aspx?proj=49409 